\title{
Review
}

\section{The role of p53 in cell metabolism}

\author{
Xing-ding ZHANG, Zheng-hong QIN, Jin WANG* \\ Department of Pharmacology and Aging and Nervous Diseases, Soochow University School of Medicine, Suzhou 215123, China
}

\begin{abstract}
The p53 tumor suppressor gene has recently been shown to mediate metabolic changes in cells under physiological and pathological conditions. It has been revealed that p53 regulates energy metabolism, oxidative stress, and amino acid metabolism through balancing glycolysis and oxidative phosphorylation (OXPHOS) as well as the autophagy pathway. p53 is activated by metabolic stress through AMP-activated protein kinase (AMPK) and the mammalian target of rapamycin (mTOR) signaling pathways. p53 regulates OXPHOS through the transcriptional regulation of fructose-2,6-bisphosophatase, TP53-induced glycolysis regulator (TIGAR) and synthesis of cytochrome $c$ oxidase (SCO2) subunit of complex IV of the electron transport chain. p53 also indirectly influences the energy metabolism through regulating glucose transporter (GLUT) expression, glutaminase 2 (GLS2) and fatty acid synthase (FAS). In addition, p53 regulates autophagy to provide cell metabolites for surviving through damage regulated autophagy modulator (DRAM1). Here we review the recent findings to elucidate the important role of p53 in cell metabolism.
\end{abstract}

Keywords: tumor suppressor 53; glycolysis; oxidative phosphorylation; TP53-induced glycolysis regulator; damage-regulated autophagy modulator

Acta Pharmacologica Sinica (2010) 31: 1208-1212; doi: 10.1038/aps.2010.151; published online 23 Aug 2010

\section{Introduction}

In the past decades, p53 is probably one of the most widely studied proteins because of its pivotal role in tumorogenesis, cell death and survival. p53 is a transcription factor that mediates cell response to various detrimental stresses through a complex signaling network ${ }^{[1]}$. When cell endures a variety of insults including DNA damage, hypoxia, and oxidative stress, p53 becomes modified, which promotes both its stabilization and translocation into the nucleus, where p53 activates the expression of genes that induce cell cycle regulation, DNA repair, senescence, and cell death ${ }^{[2]}$.

It has been found that the metabolism in cancer cells was altered. The metabolic alterations in cancer cells determine how cells respond to variable nutrient and oxygen availability and promote cell proliferation, growth and survival ${ }^{[3]}$. Recent findings indicated that p53 plays an important role in metabolic shifting in cancerous cells, suggesting a new function of p53 in regulating cell metabolism. However, how p53 regulates metabolism is complicated. In this review, we will try to elucidate the complex network of p53 regulation of different metabolic pathways.

\footnotetext{
* To whom correspondence should be addressed.

E-mail wangjin@suda.edu.cn

Received 2010-07-03 Accepted 2010-07-30
}

\section{Responses of p53 to metabolic stress}

It is well established that glucose availability directly regulates cell proliferation, a response that is mediated by activation of AMP-activated protein kinase $(\mathrm{AMPK})^{[4]}$. Activation of p53 by metabolic stress is regulated by AMPK-dependent phosphorylation and influenced by mTOR. The mTOR pathway plays a critical role in the regulation of cell proliferation, survival and energy metabolism. mTOR forms two complexes in cells, mTOR complex1 (mTORC1) and mTOR complex2 (mTORC2). Metabolic stress activates AMPK, which in turn phosphorylates and activates the tuberous sclerosis complex 2 (TSC2) protein. TSC2 exerts GTPase activity to negatively regulate GTP-binding protein, Rheb, the protein that activates mTORC $^{[5]}$ (Figure 1). mTORC1 can directly or indirectly dephosphorylate p53 on Ser15 in response to nutrient starva$\operatorname{tion}^{[6-8]}$.

p53 induces the expression of a number of p53 target genes in the IGF-1/AKT and mTOR pathways, including IGF-BP3, PTEN, TSC2, AMPK b1, Sestrins1 and 2, and REDD1 ${ }^{[9-13]}$. All these gene products negatively regulate the IGF-1/AKT and mTOR pathways in response to stress signals (Figure 1). It has recently been shown that AMPK can be phosphorylated and activated by binding to sestrin1 (Sesn1) or sestrin2 (Sesn2), both of which are p53 target genes. p53 induces the expression of Sestrin1 and Sestrin 2, which interact with the a-subunits of AMPK resulting in the phosphorylation of AMPK on Thr172. This leads to the activation of AMPK and 


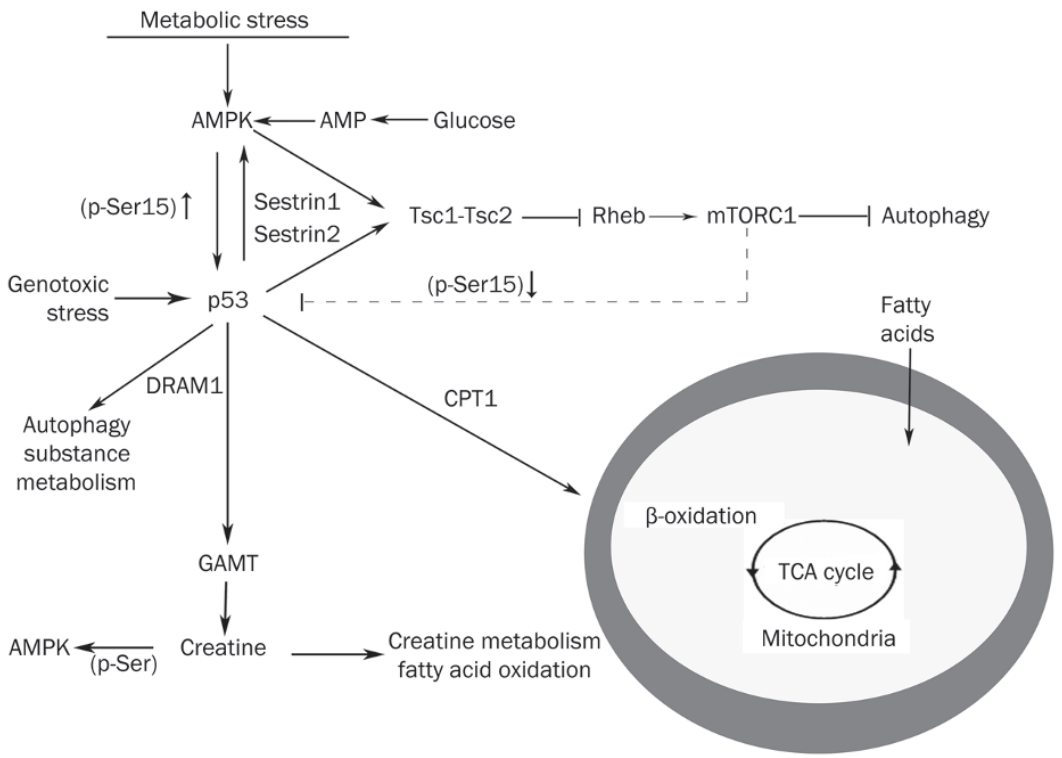

Figure 1. The AMPK-p53-mTOR pathway in metabolic adaptation. Activation of $\mathrm{p} 53$ by metabolic stress is regulated by AMP-activated protein kinase (AMPK)dependent phosphorylation and influenced by mammalian target of rapamycin complex1 (mTORC1). p53 further induces AMPK (both directly and indirectly through the sestrins) and activates the expression of tuberous sclerosis complex 2 (TSC2), resulting in the inhibition of mTOR. The activation of p53 may also cause upregulation of carnitine palmitoyltransferase (CPT1) and enhancement of $\beta$-oxidation of fatty acids. p53 regulates expression of damage-regulated autophagy modulator 1 (DRAM1), which in turn induces autophagy. p53 upregulates guanidinoacetate methyltransferase (GAMT), which regulates the creatine metabolic pathway involved in fatty acid metabolism.
TSC2, and the inhibition of mTORC1 activity ${ }^{[11]}$. Sestrins have been shown to decrease intracellular reactive oxygen species (ROS), probably by regenerating over-oxidized peroxiredoxins that deoxidize $\operatorname{ROS}^{[14-16]}$. Sesn1 and Sesn2 expression, elevated by $\mathrm{p} 53$ in response to genotoxic stress, has been shown to be responsible for the resistance to oxidative stress induced by p53 both in vitro and in vivo ${ }^{[14,17,18]}$. It appears that Sesn1/2 interact with the a-catalytic subunits of AMPK. This interaction increases AMPK phosphorylation and induces its activity. Moreover, Sesn1/2 preferentially activate AMPK toward the phosphorylation of TSC2, and they also bind TSC2, directing AMPK to TSC2 ${ }^{[11]}$. The results presented by Budanov and Karin imply that metabolic stress in the presence of p53 should have a more substantial inhibitory effect on mTORC1 than in the absence of $\mathrm{p} 53^{[11]}$. Thus, through the transcription of seven different target genes, p53 negatively regulates the IGF-1/AKT and mTOR pathways, creating an inter-pathway network that permits cells under stress to shut down cell growth and division to avoid the introduction of errors during these processes.

\section{The role of p53 in regulating glycolysis}

Glycolysis is the metabolic pathway that converts glucose into pyruvate and to produce ATP and NADH. Glycolysis exists in almost all species, which indicates that it is one of the most ancient known metabolic pathways ${ }^{[19]}$. Recently, p53 was found to regulate glycolysis via regulating expression of fructose-2,6-bisphosphatase, TP53-induced glycolysis regulator $(\text { TIGAR })^{[20,21]}$. TIGAR down-regulates glycolysis by degrading fructose-2,6-bisphosphate (Fru-2,6- $\mathrm{P}_{2}$ ), a potent allosteric effector of glycolytic enzyme 6-phosphofructo-kinase-1 (PFK-1) ${ }^{[20]}$. The expression of TIGAR not only decreases glycolytic activity by dephosphorylating Fru-2,6- $\mathrm{P}_{2}$ to Fru-6-P, but also switch glucose to an alternative pathway, the pentose phosphate pathway (PPP), along with deceasing ROS generation and apoptosis by promoting glutathione production. PPP produces more nicotinamide adenine dinucleotide phosphate
$(\mathrm{NADPH})^{[20]}$ (Figure 2). NADPH is used as a reducing agent in anabolic reactions, such as lipid and nucleic acid synthesis. Mutant p53 has also been found to activate expression of hexokinase 2 (HK2) and phosphoglycerate mutase (PGM). Both HK2 and PGM participate in the glycolysis pathway. It was demonstrated that mutant p53 up-regulates expression of HK2 gene in heptatoma cells, which suggested p53 connects loss of the cell cycle control and increased glycolysis in cancer cells $^{[22,23]}$ (Figure 2). The inhibitory effect on expression of PGM by normal p53 has been found mediated by inhibiting the expression of the glucose transporters GLUT1 and GLUT4 ${ }^{[24,25]}$. This mechanism was demonstrated to be important for immortalizing mouse embryo fibroblasts ${ }^{[24,26]}$. Through regulating glycolysis, p53 can affect cellular ROS levels, as both TIGAR and PGM show antioxidant effects ${ }^{[20,24]}$. TIGAR diverts glucose through the PPP to lower ROS levels ${ }^{[27]}$ while PGM enhances glycolysis to reduce ROS production by decreasing mitochondrial respiration ${ }^{[28]}$. p53 regulates the expression of these genes in an opposite way. It is clear that p53 can induce the expression of some antioxidant genes (such as TIGAR), repress the expression of others (such as PGM), while also activate genes that enhance oxidative stress. Taken together, both the pro-oxidant and antioxidant functions of p53 are thought to contribute to tumor suppression.

\section{The role of $\mathrm{p} 53$ in regulating oxidative phosphorylation} Oxidative phosphorylation (OXPHOS) is another energy metabolic pathway that utilizes energy released by the oxidation of nutrients through the tricarboxylic acid (TCA) cycle to produce adenosine triphosphate (ATP) with higher efficiency than glycolysis. Enzymes of the tricarboxylic acid (TCA) cycle reside in the mitochondrion, catalyzing the oxidation of pyruvate and other substrates for maximal ATP production through electron transport-coupled OXPHOS ${ }^{[29]}$ (Figure 2 ). It has been found in mice and in human cancer cells that p53 directly regulates mitochondrial oxygen consumption 


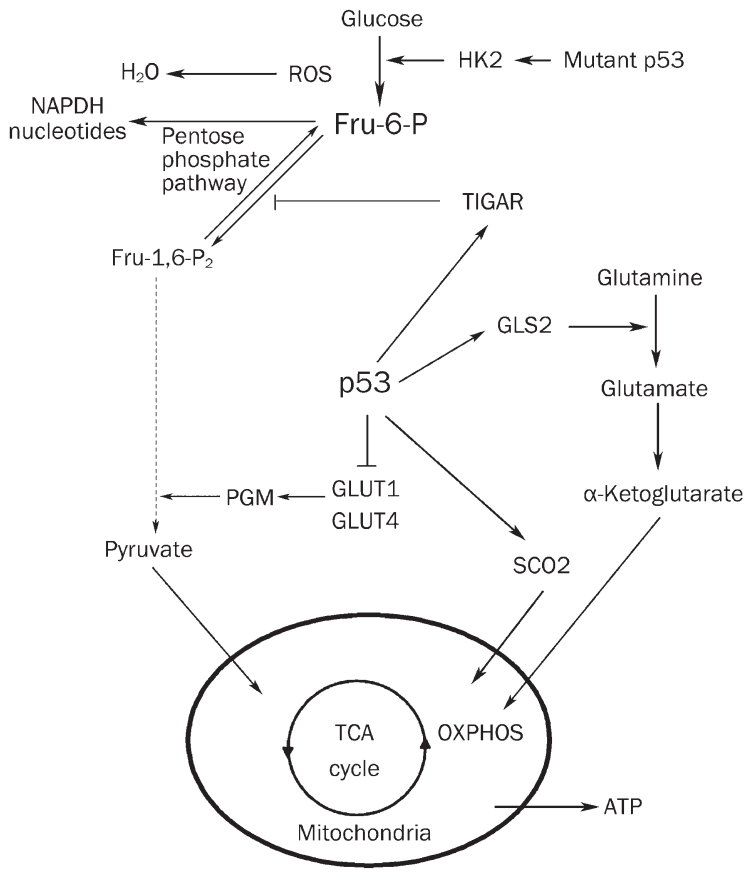

Figure 2. p53 regulation of energy metabolism. p53 regulates energy metabolism through target proteins that is directly or indirectly involved in energy metabolic pathways. p53 inhibits the expression of the glucose transporters GLUT1 and GLUT4, resulting decrease of the levels of phosphoglycerate mutase (PGM), an enzyme in glycolytic pathway, while increase the expression of TP53-induced glycolysis and apoptosis regulator (TIGAR). TIGAR diverts glucose to an alternative pathway, the pentose phosphate pathway (PPP), which leads to the production of nicotinamide adenine dinucleotide phosphate (NADPH). NADPH is used for the removal of reactive oxygenspecies (ROS). Mutant p53 has been found to activate expression of hexokinase 2 (HK2), which is an important enzyme involving in glycolysis. p53 induces GLS2 to enhance mitochondrial respiration by increasing the production of glutamate and $\alpha$-ketoglutarate. p53 targets another gene named synthesis of cytochrome c oxidase 2 ( $\mathrm{SCO} 2$ ). $\mathrm{SCO} 2$ regulates the cytochrome c oxidase complex, which is essential for mitochondrial respiration and utilization of oxygen to produce energy (ATP). The end-product of glycolysis, pyruvate, enters the tricarboxylic acid (TCA) cycle located within the mitochondria.

through an important assembly protein called synthesis of cytochrome $c$ oxidase $(\mathrm{SCO} 2)^{[21]}$. In mammalian cells, $\mathrm{SCO} 2$ is required for assembly of the cytochrome $c$ oxidase (COX) complex. COX catalyzes the transfer of reducing equivalents from cytochrome $c$ to molecular oxygen and pumps protons across the inner mitochondrial membrane. SCO2 is conserved in organisms as diverse as yeast and humans. p53 in normal tissue induces $\mathrm{SCO} 2$ expression, which ensures the maintenance of the COX and enhances mitochondrial respiration ${ }^{[21]}$. The decreased oxidative phosphorylation activity in p53-deficient cells can be restored by expressing exogeneous $\mathrm{SCO} 2$ at physiologic level ${ }^{[30]}$. In human colon cancer cell lines, DLD1 and SW480, the expression of SCO2 can increase oxygen consumption in the presence of p53 mutations ${ }^{[21]}$. In HCT116 human colon cancer cell line, deficiency in p53 causes low expression of SCO2, resulting in lower OXPHOS which is balanced by the increase in glycolysis ${ }^{[30]}$. This suggests that the downregulation of p53-dependent regulation of $\mathrm{SCO} 2$ impairs the mitochondrial respiratory chain, causing a shifting of ATP production from OXPHOS to glycolysis.

In addition to SCO2, glutaminase 2 (GLS2) is a newly identified p53- regulated protein ${ }^{[31]}$, which encodes a mitochondrial glutaminase that catalyzes the hydrolysis of glutamine to glutamate. GLS2 regulates cellular energy metabolism by increasing the production of glutamate anda-ketoglutarate, that in turn, results in enhanced mitochondrial respiration and ATP generation (Figure 2).

\section{p53 and the Warburg effect}

The Warburg effect is a theory first postulated by the Nobel laureate Otto Heinrich Warburg to explain the connection between malignant tumor growth and altered energy metabolism. The fact that the needed energy for malignant tumor growth is supplied mainly by non-oxidative pathway, glycolysis $^{[32]}$ is in contrast to that of normal cells which mainly generate energy from oxidative breakdown of pyruvate, an end-product of glycolysis, within the mitochondria. Warburg found a fundamental difference between normal and cancerous cells to be the ratio of glycolysis to respiration, which is known as the Warburg effect. The recent found evidence that deficiency of p53 in cancer cells upregulates glycolysis and at the same time down-regulates OXPHOS in cancer cells suggesting that $\mathrm{p} 53$ status plays a pivotal role in the underlying mechanism for the Warburg effect (Figure 2).

These findings together link the p53 protein with energy metabolism, and provide a novel mechanism that contributes to the Warburg effect, and also suggest a novel mechanism of p53 in tumor suppression via metabolic regulation. Considering the importance of p53 in tumor suppression and the high mutation rate of p53 $(>50 \%)$ in human tumors, these findings suggest that the mutation of the p53 gene and the resultant loss of function of the $\mathrm{p} 53$ protein in tumors could be an important genetic change contributing to the Warburg effect (Figure 2).

\section{The role of p53 in fatty acid metabolism}

Fatty acids are important metabolic intermediates. Besides being used for lipid synthesis and protein modification (eg palmitoylation, myristoylation, and synthesis of glycerophosphatidylinositol anchors), fatty acids can be degraded through mitochondrial $\beta$-oxidation, to supply substrates for oxidative phosphorylation to generate ATP. p53 can regulate fatty acid metabolism through guanidinoacetate methyltransferase (GAMT), which regulates the creatine metabolic pathway involved in fatty acid metabolism ${ }^{[33]}$ (Figure 1). GAMT converts guanidineacetate to creatine that is linked to energygenerating pathways that play an essential role in the regulation of ATP homeostasis. In cancer cells, energy metabolism is altered, which includes the fatty acid biosynthesis pathway through fatty acid synthase (FAS). FAS is a multifunctional enzyme that performs a series of sequential reactions to convert acetyl-CoA and malonyl-CoA to palmitate ${ }^{[34]}$. It is evident 
that expression of FAS and abnormally active endogenous fatty acid synthetic metabolism are elevated in many human cancers, including carcinomas of breast, prostate, endometrium, and colon ${ }^{[3,36]}$. GAMT is linked to FAS by the observation that upon treatment of cells with creatine, phosphorylation of AMPK and acetyl-CoA carboxylase (ACC) is increased, which indicates that FAS has been switched on $^{[32]}$. Activation of the p53 by metabolic stress also enhances $\beta$-oxidation of fatty acids ${ }^{[37]}$ (Figure 1 ). $\beta$-oxidation is the process by which fatty acids are broken down in mitochondria and/or in peroxisomes to generate Acetyl-CoA, the entry molecule for the Krebs cycle. The induction of fatty acid $\beta$-oxidation has been reported as a marker of metabolic reprogramming in response to glucose deprivation ${ }^{[38]}$. p53 stimulates $\beta$-oxidation through the action of the carnitine palmitoyltransferase (CPT1) ${ }^{[39]}$. CPT1, also known as carnitine acyltransferase 1 , is a mitochondrial enzyme located on the outer membrane of mitochondria. CPT1 initiates the import of fatty acids by binding them to carnitine. This enzyme can be inhibited by malonyl CoA.

\section{The role of p53 in autophagy pathway}

Autophagy is a major cellular pathway for the degradation of long-lived proteins and cytoplasmic organelles in eukaryotic cells to provide amino acids for cell survival when nutrients are scarce. Autophagy is a membrane trafficking process that mediates the delivery of cytoplasmic constituents to the lysosome for degradation ${ }^{[40]}$. Recent studies have shown that cytoplasmic p53 can play a direct role in the inhibition of autophagy $^{[41]}$ (Figure 1), which can be induced in response to nutrient deprivation and provides ATP that can prolong viability ${ }^{[42]}$. On the other hand, p53 can also activate autophagy preceding the apoptotic process ${ }^{[33]}$. The process of autophagy is regulated by p53 through the pathway of mTOR and damage-regulated autophagy modulator 1 (DRAM1) ${ }^{[9,44]}$.

Genotoxic stress stimulates p53-dependent up-regulation of DRAM1, a p53 target gene that encodes a lysosomal protein that induces autophagy ${ }^{[44]}$. It has been found that p53 induces successful autophagy in a DRAM1-dependent manner ${ }^{[44]}$. It has also been demonstrated that the p53 specific inhibitor PFT effectively blocks the 3-NP-induced induction of DRAM1, LC3-II and striatal cell death ${ }^{[43]}$. In the absence of DRAM1, GFP-LC3 was diffuse within the cytoplasm with occasional puncta representing the basal level of autophagosomes within the cell. Upon DRAM1 induction, a marked increase in the presence of GFP-LC3 puncta was observed, indicating a clear role for DRAM1 and p53 in the regulation of autophagy ${ }^{[44]}$. The mechanisms through which DRAM1 causes the formation of autophagosomes are not clear. It may be that DRAM1 sends a feedback signal to proteins that are involved in the initiation of autophagy or DRAM1 works in conjunction with other factors downstream of $\mathrm{p} 53^{[45]}$. Besides DRAM1, the activation of p53 inhibits mTOR activation and regulates its downstream targets, including autophagy ${ }^{[46]}$. mTOR appears to directly or indirectly affect the Atg proteins, resulting in interference with the formation of autophagosomes ${ }^{[47]}$.

\section{Conclusion and future directions}

In summary, as well as having well-defined roles in cell cycle regulation and apoptosis, recent studies have shown that p53 regulates stress-induced transcriptional programs that function to regulate pathways of energy metabolism. p53 has been shown to respond to metabolic changes and to influence metabolic pathways through several mechanisms. It became clear that tumor cells depend on metabolic alterations for their continued growth and survival. The activation of p53 to regulate several aspects of metabolism, including glycolysis, autophagy, OXPHOS and mTOR pathway, reveals that there are functions for p53 in the regulation of other metabolic diseases.

\section{References}

1 Levine A. p53, the cellular gatekeeper for growth and division. Cell 1997; 88: 323-31.

2 Vogelstein B, Lane D, Levine A. Surfing the p53 network. Nature 2000; 408: 307-10.

3 Gottlieb E, Vousden KH. p53 Regulation of metabolic pathways. Cold Spring Harb Perspect Biol 2010; 2: a001040.

4 Hardie DG. The AMP-activated protein kinase pathway - new players upstream and downstream. J Cell Sci 2004; 117: 5479-87.

5 Inoki K, Zhu T, Guan K. TSC2 mediates cellular energy response to control cell growth and survival. Cell 2003; 115: 577-90.

6 Feng Z, Jin S, Zupnick A, Hoh J, de Stanchina E, Lowe S, et al. p53 tumor suppressor protein regulates the levels of huntingtin gene expression. Oncogene 2006; 25: 1-7.

7 Jones R, Plas D, Kubek S, Buzzai M, Mu J, Xu Y, et al. AMP-activated protein kinase induces a p53-dependent metabolic checkpoint. Mol Cell 2005; 18: 283-93.

8 Imamura K, Ogura T, Kishimoto A, Kaminishi M, Esumi H. Cell cycle regulation via p53 phosphorylation by a $5^{\prime}$-AMP activated protein kinase activator, 5-aminoimidazole- 4-carboxamide-1-beta$D$-ribofuranoside, in a human hepatocellular carcinoma cell line. Biochem Biophys Res Commun 2001; 287: 562-7.

9 Feng $\mathrm{Z}$, Zhang $\mathrm{H}$, Levine $\mathrm{AJ}$, Jin $\mathrm{S}$. The coordinate regulation of the p53 and mTOR pathways in cells. Proc Natl Acad Sci USA 2005; 102: 8204-9.

10 Feng Z, Hu W, de Stanchina E, Teresky AK, Jin S, Lowe S, et al. The regulation of AMPK $\beta 1$, TSC2, and PTEN expression by p53: stress, cell and tissue specificity, and the role of these gene products in modulating the IGF-1-AKT-mTOR pathways. Cancer Res 2007; 67: 3043-53.

11 Budanov A, Karin M. p53 target genes sestrin1 and sestrin2 connect genotoxic stress and mTOR signaling. Cell 2008; 134: 451-60.

12 Stambolic V, MacPherson D, Sas D, Lin Y, Snow B, Jang Y, et al. Regulation of PTEN transcription by p53. Mol Cell 2001; 8: 317-25.

13 Ellisen L, Ramsayer K, Johannessen C, Yang A, Beppu H, Minda K, et al. REDD1, a developmentally regulated transcriptional target of $p 63$ and p53, links p63 to regulation of reactive oxygen species. Mol Cell 2002; 10: 995-1005.

14 Budanov A, Shoshani T, Faerman A, Zelin E, Kamer I, Kalinski H, et al. Identification of a novel stress-responsive gene Hi95 involved in regulation of cell viability. Oncogene 2002; 21: 6017-31.

15 Budanov AV, Sablina AA, Feinstein E, Koonin EV, Chumakov PM. Regeneration of peroxiredoxins by p53-regulated sestrins, homologs of bacterial AhpD. Science 2004; 304: 596-600.

16 Velasco-Miguel S, Buckbinder L, Jean P, Gelbert L, Talbott R, Laidlaw J, 
et al. PA26, a novel target of the p53 tumor suppressor and member of the GADD family of DNA damage and growth arrest inducible genes. Oncogene 1999; 18: 127-37.

17 Matheu A, Maraver A, Klatt P, Flores I, Garcia-Cao I, Borras C, et al. Delayed ageing through damage protection by the Arf/p53 pathway. Nature 2007; 448: 375-9.

18 Sablina A, Budanov A, Ilyinskaya G, Agapova L, Kravchenko J, Chumakov $P$. The antioxidant function of the p53 tumor suppressor. Nat Med 2005; 11: 1306-13.

19 Romano A, Conway T. Evolution of carbohydrate metabolic pathways. Res Microbiol 1996; 147: 448-55.

20 Bensaad K, Tsuruta A, Selak M, Vidal M, Nakano K, Bartrons R, et al. TIGAR, a p53-inducible regulator of glycolysis and apoptosis. Cell 2006; 126: 107-20.

21 Matoba S, Kang J-G, Patino WD, Wragg A, Boehm M, Gavrilova O, et al. p53 regulates mitochondrial respiration. Science 2006; 312: 1650-53.

22 Bustamante E, Pedersen PL. High aerobic glycolysis of rat hepatoma cells in culture: role of mitochondrial hexokinase. Proc Natl Acad Sci USA 1977; 74: 3735-9.

23 Mathupala SP, Heese C, Pedersen PL. Glucose catabolism in Cancer Cells. The type II hexokinase promoter contains functionally active response elements for the tumor suppressor p53. J Biol Chem 1997; 272: $22776-80$.

24 Kondoh H, Lleonart ME, Gil J, Wang J, Degan P, Peters G, et al. Glycolytic Enzymes Can Modulate Cellular Life Span. Cancer Res 2005; 65: 177-85.

25 Schwartzenberg-Bar-Yoseph F, Armoni M, Karnieli E. The tumor suppressor p53 down-regulates glucose transporters GLUT1 and GLUT4 gene expression. Cancer Res 2004; 64: 2627-33.

26 Ruiz-Lozano P, Hixon ML, Wagner MW, Flores Al, Ikawa S, Baldwin AS Jr, et al. p53 Is a transcriptional activator of the muscle-specific phosphoglycerate mutase gene and contributes in vivo to the control of its cardiac expression. Cell Growth Differ 1999; 10: 295-306.

27 Tian WN, Braunstein LD, Apse K, Pang J, Rose M, Tian X, et al. Importance of glucose-6-phosphate dehydrogenase activity in cell death. Am J Physiol Cell Physiol 1999; 276: C1121-31.

28 Brand K, Hermfisse U. Aerobic glycolysis by proliferating cells: a protective strategy against reactive oxygen species. FASEB J 1997; 11: 388-95.

29 Jones RG, Thompson CB. Tumor suppressors and cell metabolism: a recipe for cancer growth. Genes Dev 2009; 23: 537-48.

30 Ma W, Sung H, Park J, Matoba S, Hwang P. A pivotal role for p53: balancing aerobic respiration and glycolysis. J Bioenerg Biomembr 2007; 39: 243-6.

31 Hu W, Zhang C, Wu R, Sun Y, Levine A, Feng Z. Glutaminase 2, a novel $p 53$ target gene regulating energy metabolism and antioxidant function. Proc Natl Acad Sci USA 2010; 107: 7455-60.
32 Warburg 0. On the origin of cancer cells. Science 1956; 123: 30914.

33 Ide T, Brown-Endres L, Chu K, Ongusaha P, Ohtsuka T, El-Deiry W, et al. GAMT, a p53-inducible modulator of apoptosis, is critical for the adaptive response to nutrient stress. Mol Cell 2009; 36: 379-92.

34 Li JN, Gorospe M, Chrest FJ, Kumaravel TS, Evans MK, Han WF, et al. Pharmacological inhibition of fatty acid synthase activity produces both cytostatic and cytotoxic effects modulated by p53. Cancer Res 2001; 61: 1493-99.

35 Alo' P, Visca P, Marci A, Mangoni A, Botti C, Di Tondo U. Expression of fatty acid synthase (FAS) as a predictor of recurrence in stage I breast carcinoma patients. Cancer 1996; 77: 474-82.

36 Swinnen JV, Esquenet M, Goossens K, Heyns W, Verhoeven G. Androgens stimulate fatty acid synthease in the human prostate cancer cell line LNCaP. Cancer Res 1997; 57: 1086-90.

37 Buzzai M, Jones RG, Amaravadi RK, Lum JJ, DeBerardinis RJ, Zhao $\mathrm{F}$, et al. Systemic treatment with the antidiabetic drug metformin selectively impairs p53-deficient tumor cell growth. Cancer Res 2007; 67: 6745-52.

38 Buzzai M, Bauer D, Jones R, Deberardinis R, Hatzivassiliou G, Elstrom $\mathrm{R}$, et al. The glucose dependence of Akt-transformed cells can be reversed by pharmacologic activation of fatty acid beta-oxidation. Oncogene 2005; 24: 4165-73.

39 DeBerardinis RJ, Lum JJ, Thompson CB. Phosphatidylinositol 3-kinase-dependent modulation of carnitine palmitoyltransferase $1 \mathrm{~A}$ expression regulates lipid metabolism during hematopoietic cell growth. J Biol Chem 2006; 281: 37372-80.

40 Xie Z, Klionsky D. Autophagosome formation: core machinery and adaptations. Nat Cell Biol 2007; 9: 1102-9.

41 Tasdemir E, Maiuri M, Galluzzi L, Vitale I, Djavaheri-Mergny M, D’Amelio $\mathrm{M}$, et al. Regulation of autophagy by cytoplasmic p53. Nat Cell Biol 2008; 10: 676-87.

42 Lum J, DeBerardinis R, Thompson C. Autophagy in metazoans: cell survival in the land of plenty. Nat Rev Mol Cell Biol 2005; 6: 439-48.

43 Zhang XD, Wang Y, Wang Y, Zhang X, Han R, Wu JC, et al. p53 mediates mitochondria dysfunction-triggered autophagy activation and cell death in rat striatum. Autophagy 2009; 5: 339-50.

44 Crighton D, Wilkinson S, O'Prey J, Syed N, Smith P, Harrison P, et al. DRAM, a p53-induced modulator of autophagy, is critical for apoptosis. Cell 2006; 126: 121-34.

45 Crighton D, Wilkinson S, Ryan K. DRAM links autophagy to p53 and programmed cell death. Autophagy 2007; 3: 72-4.

46 Meijer A, Codogno P. Regulation and role of autophagy in mammalian cells. Int J Biochem Cell Biol 2004; 36: 2445-62.

47 Levine B, Klionsky D. Development by self-digestion: molecular mechanisms and biological functions of autophagy. Dev Cell 2004; 6 : 463-77. 